\title{
Minimum Variable Connectivity Index of Trees of a Fixed Order
}

\author{
Shamaila Yousaf, ${ }^{1,2}$ Akhlaq Ahmad Bhatti $\mathbb{D}^{1},{ }^{1}$ and Akbar Ali $\mathbb{D}^{3,4}$ \\ ${ }^{1}$ Department of Sciences and Humanities, National University of Computer and Emerging Sciences, Lahore Campus, \\ B-Block, Faisal Town, Lahore, Pakistan \\ ${ }^{2}$ Department of Mathematics, University of Gujrat, Hafiz Hayat Campus, Gujrat, Pakistan \\ ${ }^{3}$ Department of Mathematics, Faculty of Science, University of Ha'il, Ha'il, Saudi Arabia \\ ${ }^{4}$ Knowledge Unit of Science, University of Management and Technology, Sialkot, Pakistan
}

Correspondence should be addressed to Akbar Ali; akbarali.maths@gmail.com

Received 13 May 2020; Accepted 29 June 2020; Published 20 July 2020

Academic Editor: Juan L. G. Guirao

Copyright (c) 2020 Shamaila Yousaf et al. This is an open access article distributed under the Creative Commons Attribution License, which permits unrestricted use, distribution, and reproduction in any medium, provided the original work is properly cited.

\begin{abstract}
The connectivity index, introduced by the chemist Milan Randić in 1975, is one of the topological indices with many applications. In the first quarter of 1990s, Randić proposed the variable connectivity index by extending the definition of the connectivity index. The variable connectivity index for graph $G$ is defined as $\sum_{v w \in E(G)}((d(v)+\gamma)(d(w)+\gamma))^{-1 / 2}$, where $\gamma$ is a nonnegative real number, $E(G)$ is the edge set of $G$, and $d(t)$ denotes the degree of an arbitrary vertex $t$ in $G$. Soon after the innovation of the variable connectivity index, its various chemical applications have been reported in different papers. However, to the best of the authors' knowledge, mathematical properties of the variable connectivity index, for $\gamma>0$, have not yet been discussed explicitly in any paper. The main purpose of the present paper is to fill this gap by studying this topological index in a mathematical point of view. More precisely, in this paper, we prove that the star graph has the minimum variable connectivity index among all trees of a fixed order $n$, where $n \geq 4$
\end{abstract}

\section{Introduction}

All the graphs that we discuss in the present study are simple, connected, undirected, and finite. For a graph $G=(V, E)$, the number $|V(G)|$ is called its order, and $|E(G)|$ is the size of $G$. Neighbor of a vertex $v \in V(G)$ is a vertex adjacent to $v$. The set of all neighbors of vertex $v$ of $G$ is denoted by $N(v)$. The number $|N(v)|$ is called the degree of a vertex, $v \in G$, and it is denoted by $d(v)$. If $d(v)=1$, then $v$ is called a pendent vertex or a leaf. A graph of order $n$ is called an $n$-vertex graph. Denote by $P_{n}$ and $S_{n}$ the $n$-vertex path graph and the $n$-vertex star graph, respectively. The class of all $n$-vertex trees is denoted by $T_{n}$. For the (chemical) graph theoretical notation and terminology that are not defined in this paper, refer to $[1,2]$.

One of the fundamental ideas in CGT (chemical graph theory) is molecular connectivity. Chemical behavior of a compound is dependent upon its structure. QSPR/QSAR (quantitative structure-property/activity relationship) studies are progressive fields of chemical research that focus on the behavior of this dependency. The quantitative relationships are mathematical models that either enable the prediction of a continuous variable (e.g., boiling point and $L C_{50}$ toxicity) or the classification of a discrete variable (e.g., sweet/bitter and toxic/nontoxic) from structural parameters. Actually, CGT has provided many topological indices that have been and are being used in QSPR/QSAR studies for predicting the physicochemical properties of chemical compounds. Topological indices are those graph invariants that found some applications in chemistry [3-6]. For further details about the topological indices and their applications, refer to $[3,7-11]$ and the references therein.

Molecules can be modeled using graphs in which vertices correspond to atoms of the considered molecules, and the edges correspond to the covalent bonds between atoms [6]. To model the heteroatom molecules, it is better to use the vertex-weighted graphs, which are the graphs whose one or more vertices are distinguished in some way from the rest of 
the vertices [12]. Let $G$ be a vertex-weighted graph with the vertex set $\left\{v_{1}, v_{2}, \ldots, v_{n}\right\}$, and let $w_{i}$ be the weight of the vertex $v_{i}$ for $i=1,2, \ldots, n$. The augmented vertex-adjacency matrix of $G$ is an $n \times n$ matrix denoted by ${ }^{a v} \mathbf{A}(G)$ and is defined as ${ }^{a v} \mathbf{A}(G)=\left[a_{i, j}\right]_{n \times n}$, where

$$
a_{i, j}= \begin{cases}1, & \text { if } v_{i} v_{j} \in E(G) \\ w_{i}, & \text { if } i=j \\ 0, & \text { otherwise. }\end{cases}
$$

The variable connectivity index $[13,14]$, proposed by Randić, for graph $G$ is defined as

$$
{ }^{1} \chi^{f}(G)=\sum_{v_{i} v_{j} \in E(G)} \frac{1}{\sqrt{\left(d\left(v_{i}\right)+w_{i}\right)\left(d\left(v_{j}\right)+w_{j}\right)}} .
$$

We associate this index's name with its inventor Randić by calling it as the variable Randić index. This index was actually introduced within the QSPR/QSAR studies of heteroatom molecules. If $G$ is the molecular graph of a homoatomic molecule, then $w_{1}=w_{2}=\cdots=w_{n}=\gamma$ (say), and hence, the variable Randić index ${ }^{1} \chi^{f}(G)$ becomes

$$
{ }^{1} \chi^{f}(G)={ }^{v} R_{\gamma}(G)=\sum_{v_{i} v_{j} \in E(G)} \frac{1}{\sqrt{\left(d\left(v_{i}\right)+\gamma\right)\left(d\left(v_{j}\right)+\gamma\right)}} .
$$

In the rest of this paper, we denote this index by ${ }^{v} R_{\gamma}$ instead of ${ }^{1} \chi^{f}$. Clearly, if we take $\gamma=0$, then the invariant
${ }^{v} R_{\gamma}(G)$ is the classical Randić index $[10,15]$. Liu and Zhong [5] showed that the variable Randic index has more flexibility in characterizing polymers, which can lead to simpler correlations with better correlative accuracy. Details about the chemical applications of the variable Randić index can be found in $[5,7,9,12,15-22]$ and related references listed therein. It needs to be mentioned here that the variable Randić index seems to have more chemical applications than the several well-known variable indices, for example, the indices considered in [23-33]. However, to the best of the authors' knowledge, mathematical properties of the variable Randić index, for $\gamma>0$, have not yet been discussed explicitly in any paper. The main purpose of the present paper is to fill this gap by studying this topological index in a mathematical point of view. Since the trees (that are the connected graphs without cycles) form an important class of graphs both in chemical graph theory as well as in general graph theory, in this paper, we study an extremal problem related to the variable Randić index of the class of trees. We prove that the star graph $S_{n}$ has the minimum variable Randić index among all trees of a fixed order $n$, where $n \geq 4$.

\section{Main Result}

To establish the main result, we prove a lemma first.

Lemma 1. If $\gamma \geq 0$ and $s, t \geq 2$, then function $f$ defined as

$$
f(\gamma, s, t)=(s+t-1) \sqrt{s+t-1+\gamma}-(s-1) \sqrt{s+\gamma}-(t-1) \sqrt{t+\gamma}-\sqrt{\frac{(s+\gamma)(t+\gamma)}{1+\gamma}}
$$

is positive-valued.

Proof. We note that the function $\partial f / \partial s$ is strictly increasing in $t$ on the interval $(1, \infty)$ because

$$
\begin{gathered}
\frac{\partial f}{\partial s}=\sqrt{s+t-1+\gamma}+\frac{s+t-1}{2 \sqrt{s+t-1+\gamma}}-\sqrt{s+\gamma}-\frac{s-1}{2 \sqrt{s+\gamma}}-\frac{\sqrt{t+\gamma}}{2 \sqrt{(1+\gamma)(s+\gamma)}} \\
\frac{\partial}{\partial t}\left(\frac{\partial f}{\partial s}\right)=\frac{3 \sqrt{(1+\gamma)(t+\gamma)(s+\gamma)}-\sqrt{s+t-1+\gamma}}{4 \sqrt{(1+\gamma)(t+\gamma)(s+\gamma)} \sqrt{s+t-1+\gamma}}+\frac{\gamma}{4(s+t-1+\gamma)^{3 / 2}}>0,
\end{gathered}
$$

where the last inequality holds because

$$
(\gamma+1)(s+\gamma)(t+\gamma) \geq(s+\gamma)(t+\gamma)>(s+\gamma)+(t+\gamma)-1 \geq s+t+\gamma-1 .
$$

Also, note that the value of the function $\partial f / \partial s$ at $t=1$ is 0 , which implies that the function $\partial f / \partial s$ is positive-valued for $t>1$, and hence, function $f$ is strictly increasing in $s$ on the interval $(1, \infty)$. Due to the identity $f(\gamma, s, t)=f(\gamma, t, s)$, function $f$ is strictly increasing also in $t$ on the interval
$(1, \infty)$. It holds that $f(\gamma, 1, t)=f(\gamma, t, 1)=0$, and hence, $f(\gamma, s, t)>0$ for all $s, t>1$ and $\gamma \geq 0$.

Transformation 1. For $n \geq 4$, let $T$ be a tree of order $n$ containing at least two nonpendent vertices. Let $u \in V(T)$ be 
a vertex of maximum degree, and let $N_{T}(u)=\left\{v, u_{1}, u_{2}, \ldots\right.$, $\left.u_{s-1}\right\}$, where $d(v) \geq d\left(u_{i}\right)$ for $i=1,2, \ldots, s-1$. Also, take $N_{T}(v)=\left\{u, v_{1}, v_{2}, \ldots, v_{t-1}\right\}$. Note that $s \geq t \geq 2$. We transform $T$ into another tree $T$ by removing the edges $v v_{1}, v v_{2}, \ldots, v v_{t-1}$ and adding the edges $u v_{1}, u v_{2}, \ldots, u v_{t-1}$.

Lemma 2. Let $T$ and $T$ be the trees defined in Transformation 1. For $\gamma \geq 0$, it holds that

$$
{ }^{v} R_{\gamma}\left(T^{\prime}\right)<{ }^{v} R_{\gamma}(T) .
$$

Proof. By using the definition of the variable Randić index, one has

$$
\begin{aligned}
{ }^{v} R_{\gamma}(T)-{ }^{v} R_{\gamma}(\dot{T})= & \sum_{i=1}^{s-1}\left[f\left(s, d\left(u_{i}\right)\right)-f\left(s+t-1, d\left(u_{i}\right)\right)\right] \\
& +\sum_{i=1}^{t-1}\left[f\left(t, d\left(v_{i}\right)\right)-f\left(s+t-1, d\left(v_{i}\right)\right)\right] \\
& +f(s, t)-f(s+t-1,1),
\end{aligned}
$$

where

$$
f(a, b)=\frac{1}{\sqrt{(a+\gamma)(b+\gamma)}} .
$$

Equation (8) gives

$$
\begin{aligned}
{ }^{v} R_{\gamma}(T)-{ }^{v} R_{\gamma}\left(T^{\prime}\right)= & \sum_{i=1}^{s-1} f\left(s, d\left(u_{i}\right)\right)\left[1-\frac{f\left(s+t-1, d\left(u_{i}\right)\right)}{f\left(s, d\left(u_{i}\right)\right)}\right] \\
& +\sum_{i=1}^{t-1} f\left(t, d\left(v_{i}\right)\right)\left[1-\frac{f\left(s+t-1, d\left(v_{i}\right)\right)}{f\left(t, d\left(v_{i}\right)\right)}\right] \\
& +f(s, t)-f(s+t-1,1) \\
= & \sum_{i=1}^{s-1} f\left(s, d\left(u_{i}\right)\right)\left(1-\frac{\sqrt{s+\gamma}}{\sqrt{s+t-1+\gamma}}\right) \\
& +\sum_{i=1}^{t-1} f\left(t, d\left(v_{i}\right)\right)\left(1-\frac{\sqrt{t+\gamma}}{\sqrt{s+t-1+\gamma}}\right) \\
& -\frac{1}{\sqrt{(1+\gamma)(s+t-1+\gamma)}}+\frac{1}{\sqrt{(s+\gamma)(t+\gamma)}} .
\end{aligned}
$$

Since the vertex $u \in V(T)$ has the maximum degree, that is, $d(u)=s \geq d(w)$ for every $w \in V(T)$ and $d(v)=t \geq d\left(u_{i}\right) \quad$ for $\quad i=1,2, \ldots, s-1, \quad$ thus, $f\left(s, d\left(u_{i}\right)\right) \geq f(s, t)$ and $f\left(t, d\left(v_{i}\right)\right) \geq f(t, s)=f(s, t)$. Hence, equation (10) yields

$$
\begin{gathered}
{ }^{v} R_{\gamma}(T)-{ }^{v} R_{\gamma}(T) \geq \frac{s-1}{\sqrt{(s+\gamma)(t+\gamma)}}\left(1-\frac{\sqrt{s+\gamma}}{\sqrt{s+t-1+\gamma}}\right) \\
+\frac{t-1}{\sqrt{(s+\gamma)(t+\gamma)}}\left(1-\frac{\sqrt{t+\gamma}}{\sqrt{s+t-1+\gamma}}\right) \\
-\frac{1}{\sqrt{(1+\gamma)(s+t-1+\gamma)}}+\frac{1}{\sqrt{(s+\gamma)(t+\gamma)}} \\
=\frac{s-1}{\sqrt{(s+\gamma)(t+\gamma)}}+\frac{t-1}{\sqrt{(s+\gamma)(t+\gamma)}}+\frac{1}{\sqrt{(t+\gamma)(s+\gamma)}} \\
-\frac{s-1}{\sqrt{(t+\gamma)(s+t-1+\gamma)}}-\frac{1}{\sqrt{(s+\gamma)(s+t-1+\gamma)}} \\
-\frac{1}{\sqrt{(1+\gamma)(s+t-1+\gamma)}} \\
=\frac{1}{\sqrt{(s+\gamma)(t+\gamma)(s+t-1+\gamma)}}[(s+t-1) \sqrt{s+t-1+\gamma} \\
\left.-(s-1) \sqrt{s+\gamma}-(t-1) \sqrt{t+\gamma}-\sqrt{\frac{(s+\gamma)(t+\gamma)}{1+\gamma}}\right] .
\end{gathered}
$$

By using Lemma 1 in (11), we get ${ }^{v} R_{\gamma}(T)-{ }^{v} R_{\gamma}(T)>0$, as desired.

Next result is a direct consequence of Lemma 2.

Theorem 1. For $n \geq 4$ and $\gamma \geq 0$, among all trees of a fixed order $n$, star graph $S_{n}$ is the unique tree with minimum variable Randic index ${ }^{v} R_{\gamma}$, which is

$$
\frac{n-1}{\sqrt{(n-1+\gamma)(1+\gamma)}} \text {. }
$$

For $\gamma \geq 0$ and small values of $n$, we calculate the variable Randic index ${ }^{v} R_{\gamma}$ of the trees of order $n$ and find that the value of this index does not exceed from

$$
{ }^{v} R_{\gamma}\left(P_{n}\right)=\frac{2}{\sqrt{(1+\gamma)(2+\gamma)}}+\frac{n-3}{2+\gamma} .
$$

This suggests the following conjecture.

Conjecture 1. For $n \geq 4$ and $\gamma \geq 0$, among all trees of a fixed order $n$, path graph $P_{n}$ is the unique tree with maximum variable Randic index ${ }^{v} R_{\gamma}$, which is

$$
\frac{2}{\sqrt{(1+\gamma)(2+\gamma)}}+\frac{n-3}{2+\gamma} \text {. }
$$

\section{Data Availability}

There are no data concerning the present study except those presented in this manuscript. 


\section{Conflicts of Interest}

The authors declare that there are no conflicts of interest.

\section{Acknowledgments}

This work was supported by the National University of Computer and Emerging Sciences, Lahore, Pakistan.

\section{References}

[1] J. L. Gross and J. Yellen, Graph Theory, CRC Press, Boca Raton, FL, USA, 2000.

[2] F. Harary, Graph Theory, Addison-Wesley, Toronto, Canada, 1969.

[3] I. Gutman and O. E. Polansky, Mathematical Concepts in Organic Chemistry, Springer-Verlag, New York, NY, USA, 1986.

[4] L. B. Kier and L. H. Hall, Molecular Connectivity in StructureActivity Analysis, John Wiley and Sons Inc., New York, NY, USA, 1986.

[5] D. Liu and C. Zhong, "Modeling of the heat capacity of polymers with the variable connectivity index," Polymer Journal, vol. 34, no. 12, pp. 954-961, 2002.

[6] N. Trinajstić, Chemical Graph Theory, CRC Press, Boca Raton, FL, USA, 1992.

[7] G. Caporossi, I. Gutman, P. Hansen, and L. Pavlović, "Graphs with maximum connectivity index," Computational Biology and Chemistry, vol. 27, no. 1, pp. 85-90, 2003.

[8] E. Estrada and D. Bonchev, "Chemical graph theory," in Discrete Mathematics and Its Applications, CRC Press, Boca Raton, FL, USA, 2013.

[9] M. Randić, "On the history of the connectivity index: from the connectivity index to the exact solution of the protein alignment problem," SAR and QSAR in Environmental Research, vol. 26, no. 7-9, pp. 523-555, 2015.

[10] M. Randić, "On characterization of molecular branching," Journal of the American Chemical Society, vol. 97, no. 23, 1975.

[11] M. Randić, "High quality structure-property regressions. Boiling points of smaller alkanes," New Journal of Chemistry, vol. 24, no. 3, pp. 165-171, 2000.

[12] M. Randić, M. Pompe, D. Mills, and S. C. Basak, "Variable connectivity index as a tool for modeling structure-property relationships," Molecules (Basel, Switzerland), vol. 9, no. 12, pp. 1177-1193, 2004.

[13] M. Randić, "Novel graph theoretical approach to heteroatoms in quantitative structure-activity relationships," Chemometrics and Intelligent Laboratory Systems, vol. 10, no. 1-2, pp. 213-227, 1991.

[14] M. Randić, "On computation of optimal parameters for multivariate analysis of structure-property relationship," Journal of Computational Chemistry, vol. 12, no. 8, pp. 970980, 1992.

[15] M. Randić, "On history of the Randić index and emerging hostility toward chemical graph theory," MATCH Communications in Mathematical and in Computer Chemistry, vol. 59, no. 1, pp. 5-124, 2008.

[16] J. C. Dearden, "The use of topological indices in QSAR and QSPR modeling," in Advances in QSAR Modeling, K. Roy, Ed., pp. 57-88, Springer, Berlin, Germany, 2017.

[17] R. G. Domenech, J. Gálvez, J. V. de Julián-Ortiz, and L. Pogliani, "Some new trends in chemical graph theory," Chemical Reviews, vol. 108, pp. 1127-1169, 2008.
[18] M. Pompe, "Variable connectivity index as a tool for solving the anti connectivityproblem," Chemical Physics Letters, vol. 404, pp. 296-299, 2004.

[19] M. Randić and S. C. Bašak, "On use of the variable connectivity index $1 \chi^{\mathrm{f}}$ in QSAR: toxicity of aliphatic ethers," Journal of Chemical Information and Computer Sciences, vol. 41, no. 3, pp. 614-618, 2001.

[20] M. Randić and M. Pompe, "The variable connectivity index $1 \chi^{\mathrm{f}}$ versus the traditional molecular descriptors: a comparative study of $1 \chi^{\mathrm{f}}$ against descriptors of CODESSA," Journal of Chemical Information and Computer Sciences, vol. 41, no. 3, pp. 631-638, 2001.

[21] M. Randić, D. Plavšić, and N. Lerš, "Variable connectivity index for cycle-containing structures," Journal of Chemical Information and Computer Sciences, vol. 41, pp. 657-662, 2001.

[22] S. Yousaf, A. A. Bhatti, and A. Ali, "On the minimum variable connectivity index of unicyclic graphs with a given order," Discrete Dynamics in Nature and Society.

[23] S. Akhter, M. Imran, and Z. Raza, "Bounds for the general sum-connectivity index of composite graphs," Journal of Inequalities and Applications, vol. 2017, no. 1, p. 76, 2017.

[24] A. Ali, Z. Du, and K. Shehzadi, "Estimating some general molecular descriptors of saturated hydrocarbons," Molecular Informatics, vol. 38, no. 11-12, 2019.

[25] D. Amić, D. Davidovic-Amić, D. Bešlo, B. Lučić, N. Trinajstić, and S. Nikolić, "The vertex-connectivity index revisited," Journal of Chemical Information and Computer Sciences, vol. 38, no. 5, pp. 819-822, 1998.

[26] A. Behtoei, "Some relations and bounds for the general first zagreb index," MATCH Communications in Mathematical and in Computer Chemistry, vol. 81, no. 2, pp. 361-370, 2019.

[27] D. Dimitrov and A. Ali, "On the extremal graphs with respect to the variable sum exdeg index," Discrete Mathematics Letters, vol. 1, pp. 42-48, 2019.

[28] S. Elumalai and T. Mansour, "On the general zeroth-order Randić index of bargraphs," Discrete Mathematics Letters, vol. 2, pp. 6-9, 2019.

[29] F. Hayat, "On generalized atom-bond connectivity index of cacti," Iranian Journal of Mathematical Chemistry, vol. 10, pp. 319-330, 2019.

[30] M. Imran, A. Q. Baig, and H. Ali, "On molecular topological properties of hex-derived networks," Journal of Chemometrics, vol. 30, no. 3, pp. 121-129, 2016.

[31] J.-B. Liu, J. Zhao, and Z.-Q. Cai, "On the generalized adjacency, Laplacian and signless Laplacian spectra of the weighted edge corona networks," Physica A: Statistical Mechanics and Its Applications, vol. 540, p. 123073, 2020.

[32] J.-B. Liu, J. Zhao, H. He, and Z. Shao, "Valency-based topological descriptors and structural property of the generalized sierpiński networks," Journal of Statistical Physics, vol. 177, no. 6, pp. 1131-1147, 2019.

[33] A. Martínez-Pérez and J. M. Rodríguez, "A unified approach to bounds for topological indices on trees and applications," MATCH Communications in Mathematical and in Computer Chemistry, vol. 82, pp. 679-698, 2019. 